\title{
.
}

\author{
Alberto Sanz Serrano*
}

\section{INDIA Y EL SISTEMA MULTILATERAL DE COMERCIO}

India fue uno de los veintitrés miembros fundadores del GATT (Acuerdo General sobre Aranceles Aduaneros y Comercio) de 1947, la base sobre la que reposa el actual sistema multilateral de comercio que administra la Organización Mundial de Comercio (OMC). Hoy en día, India es una de los actores imprescindibles para cualquier acuerdo relevante en la OMC. Su posición está repleta de matices que no hacen sino reflejar la diversidad y complejidad interna de su propia economía. India es, en efecto, un país con extensas regiones eminentemente agrícolas, donde habitan millones de agricultores y, a la vez, en el otro extremo, un país con importantes centros tecnológicos y de servicios, altamente cualificados e internacionalizados. Este artículo presenta las prioridades de India en las diferentes áreas de los acuerdos y negociaciones de la OMC.

Palabras clave: India, OMC, sistema multilateral de comercio, aranceles, productos farmacéuticos, moratoria digital.

Clasificación JEL: F13, F14.

\section{Un multilateralismo flexible}

India defiende un sistema multilateral de comercio inclusivo, basado en reglas adoptadas por consenso. Recuerda permanentemente la obligación de dar cumplimiento a los tratados y mandatos de negociación ya aprobados, muy en especial al mandato de negociación de Doha. Es, además, uno de los principales usuarios del sistema de solución de diferencias de la $\mathrm{OMC}^{1}$ y recuerda permanentemente la

\footnotetext{
* Técnico Comercial y Economista de Estado. Versión de septiembre de 2020

DOI: https:/doi.org/10.32796/bice.2020.3128.7090

1 En 26 ocasiones ha abierto casos de solución de diferencias contra medidas adoptadas por otros miembros ( 11 casos contra EE UU y 7 contra la UE). De forma simétrica, en 32 ocasiones sus medidas han sido objeto de denuncia por parte de otros miembros (UE en 11 casos y EE UU en 8).
}

necesidad de restaurar el funcionamiento del Órgano de Apelación, paralizado por EE UU desde diciembre de 2019. Previene frente a cualquier iniciativa plurilateral por considerarla una desviación de los principios de la institución. Por tal motivo, no participa en ninguna de las tres iniciativas plurilaterales lanzadas en la Conferencia Ministerial de Buenos Aires de $2017^{2}$, a diferencia de otros países en desarrollo como China, Brasil o Rusia. Tampoco ha suscrito el recientemente creado mecanismo provisional de apelación mediante arbitraje.

De manera particular, India viene planteando sistemáticamente la necesidad de dar $\square$

2 Facilitación de las inversiones para el desarrollo, comercio electrónico y regulación doméstica de los servicios. 
cumplimiento a las disposiciones de Trato Especial y Diferenciado incluidas en los mandatos de negociación y en los propios acuerdos. $\mathrm{Ha}$ rechazado vigorosamente cualquier intento de establecer categorías entre los miembros en desarrollo y exige con insistencia que en las negociaciones en curso, como es el caso de las subvenciones a la pesca, el trato especial y diferenciado sea un componente sustancial e irrenunciable. Se opone, igualmente, a abordar esta cuestión mediante soluciones específicas, caso por caso, que intenten ir dando respuesta puntual a las necesidades concretas de países en desarrollo, por considerar que el trato especial y diferenciado es un derecho irrenunciable, no una concesión puntual.

Estas prioridades, que India defiende en la OMC, no siempre están alineadas con los objetivos de apertura económica y liberalización que recurrentemente planean sobre la agenda política de India. Veamos a continuación algunas áreas de especial interés.

\section{La cuestión agrícola. Una solución definitiva a la seguridad alimentaria}

Si hubiera que señalar un área prioritaria para India en la OMC, esta sería la de la cuestión agrícola y, más en concreto, su petición de flexibilizar las normas que limitan los programas de gasto agrícola sobre cultivos básicos.

En efecto, el Acuerdo de Agricultura de la OMC, negociado en la Ronda Uruguay, es percibido por India como un rígido corsé que impide la flexibilidad necesaria para incrementar su gasto agrícola. El acuerdo permite a India un nivel de ayuda del $10 \%$ sobre el valor de la producción. Este nivel de mínimis permitido es común para los países en desarrollo, salvo para aquellos que negociaron porcentajes diferentes en el momento de su adhesión, como China (8,5\%). En la Conferencia Ministerial de Bali (2013) se acordó, además, que si un miembro en desarrollo llevara a cabo programas públicos de constitución de stocks con fines de seguridad alimentaria que sobrepasaran este nivel, el resto de miembros se abstendría de denunciar formalmente estas medidas ante el Órgano de Solución de Diferencias, siempre y cuando dicho gasto se notificara, se limitara a programas ya existentes y no afectara a la seguridad alimentaria de otros miembros.

La situación actual de pandemia está ejerciendo en muchos países una elevada presión para rediseñar sus programas de apoyo a la agricultura, haciendo uso de las diversas flexibilidades existentes en la OMC. Así, en marzo de 2020, India ha sido el primer miembro de la OMC en acogerse a la cláusula de paz de Bali y notificar a la OMC haber superado su techo de gasto para el cultivo de arroz. Estados Unidos, que ya había presentado en la OMC una contranotificación en 2018 sobre el mecanismo de apoyo que India otorga al arroz, entre otros cultivos, se verá así privado de poder denunciar los programas notificados ante el Órgano de Solución de Diferencias.

Los miembros de la OMC, en su conjunto, vigilan que este primer uso de la cláusula de paz no dé lugar a una carrera para elevar indebidamente el apoyo interno a la agricultura. En particular, Pakistán sigue de cerca que los stocks adquiridos mediante tal excepción no terminen siendo dedicados a la exportación. La cuestión reabrirá, sin duda, el debate sobre la insuficiente transparencia en las notificaciones de gasto agrícola que los miembros deben presentar regularmente ante la OMC.

La existencia de millones de agricultores de baja renta genera no solo una gran presión política para la concesión de subsidios $\triangleright$ 
agrícolas, sino también una alta demanda de protección de las importaciones agrícolas. Así, el arancel medio ponderado aplicado por India sobre las importaciones agrícolas se eleva a un considerable $60,7 \%$, frente al $12,5 \%$ de China, el $9,2 \%$ de la UE o el $4,6 \%$ de EE UU ${ }^{3}$. Con semejante nivel de protección arancelaria no es de extrañar que las importaciones agrícolas solo representen en India un 4\% del conjunto de importaciones de mercancías.

Incluso con esta elevada protección arancelaria resulta habitual que India aplique también otras medidas de protección como las licencias o los contingentes. Tal es el caso actualmente de las leguminosas, importante fuente de proteínas, para las que India aplica cuotas muy restrictivas que varían en función de la situación del mercado, creando grandes dificultades a países exportadores.

India defiende igualmente la creación en la OMC de un mecanismo especial de salvaguardia agrícola (SSM) que permita a los países en desarrollo elevar sus aranceles agrícolas cuando se produzca un aumento de las importaciones o se registren caídas de los precios. Este mecanismo sería adicional a las salvaguardias ya existentes bajo el Acuerdo GATT (que requieren demostrar la existencia de daño) y las salvaguardias agrícolas disponibles para aqueIlos miembros de la OMC que en la Ronda Uruguay se comprometieron a transformar las restricciones cuantitativas en aranceles, que no es el caso de India.

Esta visión proteccionista del comercio agrícola ha chocado en numerosas ocasiones con otros socios. Recordemos, por ejemplo, la Conferencia Ministerial de julio de 2008, que estuvo muy cerca de cerrarse con un paquete de

3 OMC Perfiles arancelarios 2020. https://www.wto.org/english/res_e/ booksp_e/tariff_profiles20_e.pdf medidas tanto agrícolas como industriales. Sin embargo, el desencuentro final entre India y EE UU en lo referente a los subsidios agrarios y la cláusula SSM impidieron alcanzar un acuerdo. Este fracaso fue percibido por muchos como el inicio del fin de la Ronda Doha, que, desde entonces, ha sido incapaz de producir nuevos acuerdos sustanciales y se ha ido desmembrando en iniciativas de reducida ambición. India, por último, apoyó la eliminación de los subsidios a las exportaciones agrícolas adoptada en Nairobi (2015), al tratarse de un interés ofensivo más que defensivo.

Para cerrar este apartado sobre agricultura es necesario hacer referencia también al argumento que India viene sosteniendo en la OMC (junto con China), según el cual el Acuerdo de Agricultura de la Ronda Uruguay ha creado un desequilibrio de partida que impide cualquier negociación agrícola. Sostienen ambas potencias, apoyadas por muchos países en desarroIlo, que los países desarrollados obtuvieron un tratamiento privilegiado, dado que el Acuerdo de Agricultura les permite conceder ayudas agrícolas (las ayudas de caja verde y azul, por ejemplo) que no se incluyen dentro de los niveles de mínimis. Se trata, sostienen, de un privilegio que no está al alcance de los países en desarrollo.

Olvida esta crítica, no obstante, que fueron precisamente los países desarrollados los que se comprometieron a reducir sus ayudas en la Ronda Uruguay y que, además, se les asignó un nivel de mínimis del $5 \%$, inferior al $10 \%$ que se aplica a los países en desarrollo. Sea o no justificable la crítica planteada por India, lo cierto es que la controversia sobre la existencia de asimetrías históricas en el Acuerdo de Agricultura impide configurar un paquete equilibrado sobre ayuda interna a la agricultura, aspecto clave para cualquier negociación de $D$ 
calado en la OMC. Condicionar cualquier avance a la solución previa de estas asimetrías seguirá bloqueando cualquier posibilidad de cerrar acuerdos.

\section{La protección industrial}

India cuenta también con una elevada protección arancelaria sobre productos industriales. El arancel que aplica sobre las importaciones de productos industriales asciende al $14,1 \%$, en promedio del conjunto de partidas arancelarias, un tipo superior al de la UE $(4,2 \%)$, EE UU $(3,1 \%)$, China $(6,5 \%)$, Brasil $(13,9 \%)$ o Rusia $(6,1 \%)^{4}$. Además, el arancel consolidado en las listas GATT que puede aplicar India es muy superior, del $36 \%$ en promedio, lo que le concede un gran margen para aumentar los aranceles aplicados, dentro de los topes permitidos.

Con tal nivel de protección, India no apoya retomar las negociaciones de acceso a mercado para productos no agrícolas, completamente inactivas desde la ya comentada conferencia de julio de 2008.

El caso de los aranceles sobre los teléfonos móviles puede servir muy bien para ilustrar las dificultades que afronta India en esta materia arancelaria. Suscribió, en 1996, el Acuerdo de las Tecnologías de la Información (ITA). Este acuerdo eliminó de manera progresiva los aranceles sobre un amplio conjunto de bienes tecnológicos, como ordenadores, teléfonos o impresoras entre los veintinueve países signatarios, beneficio que se extiende a todos los demás miembros de la OMC, en virtud de la cláusula de nación más favorecida. El acuerdo

4 OMC Perfiles arancelarios 2020. https://www.wto.org/english/res_e/ booksp_e/tariff_profiles20_e.pdf se alcanzó justo al inicio de la era de internet y supuso un claro impulso al desarrollo del comercio de productos informáticos en décadas posteriores. China, por aquel entonces, no era todavía miembro de la OMC, lo que permitía a India seguir aplicando elevados aranceles en su comercio bilateral con China. Ahora bien, con la adhesión de China a la OMC en 2001 se acordó su integración en el ITA, eliminándose así los aranceles entre ambas potencias para estos productos. Sin posibilidad de fijar aranceles, la poderosa competencia de China es percibida por India como un obstáculo al desarroIlo de su propia industria tecnológica, incluida la fabricación de teléfonos móviles.

India buscó entonces formas de reintroducir los aranceles sobre algunos bienes incluidos en el ITA. Así, con ocasión de las redefiniciones periódicas de los códigos arancelarios para adaptarlos al sistema armonizado de la Organización Mundial de Aduanas, India fue reasignando los teléfonos móviles a otros códigos para liberarlos del compromiso de eliminación del arancel. Argumenta que se trata de una reasignación basada en criterios puramente técnicos, en la medida en que los teléfonos móviles que existían en 1996 no son comparables a los teléfonos inteligentes de hoy en día, que, en su opinión, no estaban cubiertos por el ITA. Sea como fuere, lo cierto es que tanto la UE como Japón y Taiwán han abierto recientemente casos de solución de diferencias contra esta modificación arancelaria. China, principal productor de móviles del mundo, sin embargo, no ha abierto una disputa dado que mantiene como práctica no denunciar nunca formalmente a países en desarrollo. Desencantada por los problemas creados por el ITA de 1996, India no formó parte en el Acuerdo ITA2, que en el año 2016 amplió la lista de productos tecnológicos sin arancel. 
Este caso no es sino un ejemplo de la defensa permanente que India viene realizando en Ginebra para mantener espacios de maniobra en su política industrial. La situación es común a muchos otros sectores. En los últimos años, haciendo uso del amplio margen entre tipos efectivos y tipos máximos consolidados, la Administración Modi elevó los aranceles sobre productos industriales tales como equipos médicos, calzado o muebles, y permanece siempre atenta a posibles incrementos de importaciones que supongan una amenaza a su planes de desarrollo industrial. En ocasiones, estas subidas arancelarias van destinadas exclusivamente a determinados países. Estados Unidos, por ejemplo, abrió un caso de solución de diferencias frente a la India por este tipo de aranceles que no respetan la cláusula de nación más favorecida. Más recientemente, en 2020, India subió sus aranceles sobre los paneles fotovoltaicos producidos en China.

Cabe señalar asimismo que, más allá del caso del ITA ya mencionado, India no ha sido firmante de ningún acuerdo plurilateral de eliminación de aranceles, como el acuerdo de aeronaves civiles o el de bienes farmacéuticos, ni asumió las horquillas de reducción arancelaria para productos químicos negociadas por una decena de miembros durante la Ronda Uruguay. Tampoco participó en las negociaciones plurilaterales para reducción de aranceles del Acuerdo de Bienes Medioambientales (EGA), paralizadas en 2016.

Así pues, la posición de India, contraria a retomar en la OMC discusiones sobre aranceles industriales, dificulta la configuración de un paquete atractivo de negociaciones en la organización que, además de cuestiones del comercio de productos agrícolas, contenga elementos de interés para el conjunto de miembros de la OMC.

\section{La producción de medicamentos bajo el TRIP}

Otra área de especial relevancia para India es el de los productos farmacéuticos. Con una producción anual de unos 40.000 millones de dólares, la mitad es destinada a la exportación, en particular medicamentos genéricos. India figura entre los principales fabricantes y exportadores mundiales de paracetamol e hidroxicloroquina, medicamentos ampliamente empleados en la actual pandemia.

En India, la ley de patentes de 1970 no reconocía la posibilidad de patentar medicamentos, lo que favoreció durante los años ochenta una auténtica explosión en la producción de medicamentos genéricos, mediante técnicas de ingeniería inversa. El Acuerdo de los Derechos de Propiedad Intelectual relacionados con el Comercio (TRIPS) de la OMC obligó a India a adoptar un sistema de patentes sobre los medicamentos, que otorgue al menos veinte años de protección. Ahora bien, para no limitarse a producir medicamentos con patentes expiradas, India se acoge a diversas flexibilidades. En alguna ocasión se ha opuesto a reconocer patentes sobre mejoras en medicamentos, que pretendían perennizar la patente sin avances terapéuticos significativos. En otros casos, India ha promovido la producción de medicamentos patentados bajo las denominadas licencias obligatorias, modalidad permitida por motivos de interés público bajo el Acuerdo TRIPS, cuando no haya sido posible obtener un acuerdo con el detentor de la patente. India ha sido también uno de los principales impulsores para la modificación del Acuerdo TRIPS, que a partir de 2017 permite hacer uso de estas licencias obligatorias no solo para el mercado doméstico, sino también para la exportación a terceros países que no tengan capacidad $D$ 
de producción, lo que es percibido por India como una oportunidad comercial, también en la actual pandemia. Conviene aclarar que bajo las licencias obligatorias sigue existiendo el deber de remunerar al titular de la patente.

En la actual situación de pandemia, muchos países en desarrollo están planteándose si las flexibilidades existentes bajo el TRIPS serán suficientes para poder acceder, en poco tiempo y a un precio asequible, a los tratamientos y vacunas que vayan apareciendo. Si no fuera el caso, algunos académicos sugieren la posibilidad de acogerse a la excepción por motivos de seguridad ante situaciones excepcionales de emergencia contenida en el artículo 73 del TRIPS, que permitiría ir más allá de las licencias obligatorias ya existentes, en particular, en lo referente a la obligación de remuneración. Esta excepción, de ser invocada, supondría la apertura de un nuevo frente de disputas en la OMC, que demostraría que la excepción de seguridad nacional, planteada por EE UU en diferentes casos estos últimos años, bien podría alimentar similares peticiones por parte de otros países en diferentes áreas de la OMC.

\section{El comercio electrónico y la moratoria digital}

India es un actor muy relevante en el campo de desarrollos digitales. Su tradición matemática, la amplia oferta de programadores informáticos y la extensión en el uso del inglés hacen de India un centro internacional en el desarrollo de estas tecnologías. No se puede cerrar este resumen sin hacer mención a la cuestión del comercio electrónico en la OMC y, más en particular, a la cuestión de la moratoria digital.

Al igual que en el campo industrial y agrícola, India se muestra muy reticente a avanzar en negociaciones que regulen el comercio electrónico internacional y las transmisiones digitales internacionales. A diferencia de China, India no participa en la iniciativa plurilateral de comercio electrónico surgida en 2017 y sigue defendiendo como única vía las discusiones en el marco del Programa de Comercio Electrónico de 1998. Este programa, que no ha producido frutos tangibles en más de veinte años de discusiones, es el foro en el que India ha planteado en estos últimos años el debate sobre la posibilidad de aplicar aranceles sobre las transmisiones electrónicas, una cuestión de gran calado conceptual y de imprevisibles consecuencias.

Para explicarlo hay que remontarse a la decisión adoptada en la Conferencia Ministerial de Ginebra de 1998, según la cual los miembros de la OMC se abstendrían, durante un periodo de dos años, de aplicar aranceles (gravámenes en aduana) sobre las transmisiones electrónicas. En ese momento, los albores del boom de internet y de las comunicaciones electrónicas, ya se percibía que muchos bienes físicos podían ser sustituidos por transmisiones electrónicas que escaparían a los aranceles. Un libro digital, por ejemplo, no podría ser gravado con aranceles, a diferencia de un libro físico. Este compromiso para no imponer aranceles sobre las transmisiones electrónicas se ha venido renovando cada dos años en la OMC y se conoce como la moratoria sobre el comercio electrónico o, simplemente, como la moratoria digital.

India y Sudáfrica desean forzar un debate en la OMC para delimitar el ámbito de dicha moratoria. Los términos de la misma resultan ambiguos, como muchos otros acuerdos en la OMC. Para algunos, la moratoria se limitaría a aquellos bienes que se pueden digitalizar (un libro, un disco o una película de cine); para otros, se refiere a las transmisiones electrónicas $\square$ 
puramente digitales desde su concepción (por ejemplo, las transmisiones para impresión 3D, los flujos de datos en internet, los productos de streaming, etc.).

Cualquiera que sea la posición que adoptemos en este debate, las consecuencias pueden ser muy significativas. Si entendiéramos que el ámbito de aplicación de la moratoria es estrecho, estaríamos dando a entender que existe gran margen para la fijación de aranceles sobre el resto de transmisiones electrónicas, incluso bajo la actual moratoria. $\mathrm{Si}$, por el contrario, entendiéramos que la moratoria actual cubre una amplia gama de transmisiones, entonces estaríamos considerando que una eventual eliminación de la misma permitiría gravar un amplio conjunto de transmisiones electrónicas.

Todo ello sin tener en cuenta la discusión sobre si las transmisiones electrónicas son bienes o servicios. Si consideramos que se trata de bienes digitalizados, entonces podríamos encontrar argumentos para aplicar aranceles. Si entendiéramos, por el contrario, que las transmisiones electrónicas se encuentran en el terreno de los servicios, abriríamos el debate sobre si el Acuerdo General sobre el Comercio de Servicios (GATS) permite la aplicación de aranceles, una provocación conceptual de imprevisibles consecuencias.

Un informe de la Conferencia de las Naciones Unidas sobre Comercio y Desarrollo (UNCTAD) ${ }^{5}$ cifra en 10.000 millones de dólares la pérdida de recaudación arancelaria derivada de la moratoria. La Organización para la Cooperación y el Desarrollo Económico (OCDE) $)^{6}$, por su parte, ha señalado que los beneficios de la misma superan con creces la pérdida de recaudación

https://unctad.org/en/PublicationsLibrary/ser-rp-2019d1_en.pdf 6 http://www.oecd.org/trade/uqbar-orbis-and-the-wto-moratorium/ y sugiere soluciones a través de la imposición interna, no mediante la creación de aranceles.

Con este debate, India está dejando entrever que puede oponerse a la renovación de la moratoria digital en la próxima Conferencia Ministerial prevista para junio de 2020 en Kazajstán. El fin de esta moratoria no tendría consecuencias inmediatas, dadas las limitaciones técnicas que existen para captar y gravar las transmisiones electrónicas internacionales, pero, con el tiempo, los avances tecnológicos permitirían un control fiscal de estas transmisiones, abriendo así la vía a un nuevo proteccionismo digital, de imprevisibles consecuencias.

Cabe señalar que la próxima Conferencia Ministerial deberá adoptar una decisión no solo sobre la renovación de la moratoria digital aquí comentada, sino también sobre otra moratoria, también bienal, que fija una cláusula de paz ante ciertos incumplimientos en el ámbito del Acuerdo TRIPS. Si India pusiera en cuestión la renovación de la moratoria digital, no sería de extrañar que otros miembros de la OMC pusieran en cuestión la prórroga de la moratoria TRIPS, una flexibilidad que puede resultar esencial para explotar todas las flexibilidades del Acuerdo TRIPS en estos momentos de pandemia.

Lo que está en juego es la posibilidad de que el mundo digital que ahora estrenamos pueda estar sujeto a los mismos aranceles que se vienen aplicando desde hace siglos sobre las mercancías. Las limitaciones a la expansión de las tecnologías y el conocimiento que tal escenario provocaría nos llevan a pensar que lo más sensato no es gravar las transacciones digitales en el momento de su transmisión transfronteriza, sino que lo que urge es adaptar el sistema fiscal a la rápida evolución de la economía digital para corregir las posibles lagunas normativas o recaudatorias que se puedan presentar. 


\section{Reglas para el siglo xxI}

Hemos visto cómo, en el contexto actual de fuertes tensiones y caídas drásticas de los flujos internacionales de comercio e inversión, India y otros muchos países en desarrollo desean contar con flexibilidades adicionales en la OMC que les otorguen un mayor margen de maniobra para sus políticas internas. Es el caso del gasto agrícola, la protección a la industria, las patentes sobre los medicamentos o la economía digital, que hemos analizado.

Ahora bien, aun siendo comprensible la demanda de flexibilidades a corto plazo para hacer frente a la gravedad de la situación creada por la pandemia, la Conferencia Ministerial de la OMC de 2021 debería ser capaz de lograr pequeños avances que, al menos, eviten males mayores. India, por su condición de gran actor y país en desarrollo, bien podría jugar un papel esencial en el éxito de esta conferencia. Para ello sería necesario, no obstante, que las flexibilidades que solicita en las diversas áreas no fueran entendidas como desviaciones permanentes, sino como instrumentos temporales para poder cumplir con los acuerdos. Un enfoque pragmático y no ideológico sobre la cuestión del trato especial y diferenciado resulta imprescindible.

Tampoco es realista pensar que se pueda abrir una nueva etapa en el pilar negociador de la OMC si todos los miembros no son capaces de permitir una agenda de negociaciones equilibrada, que, con menor ambición y complejidad que la Ronda Doha, contenga intereses ofensivos y defensivos para los diferentes miembros. La cuestión agrícola no debe ser vista como un prerrequisito para otras discusiones. Para poder involucrar a todos los actores es necesario retomar discusiones sobre protección industrial y desarrollar un marco más riguroso para los subsidios industriales, que ponga coto a prácticas anticomerciales no contempladas en el actual Acuerdo de Subvenciones y Medidas Compensatorias de la OMC.

Por último, resultaría muy positivo que India se pudiera unir a las iniciativas plurilaterales en marcha, en especial, la de comercio electrónico, por su mayor calado. En cualquier caso, siempre es mejor participar en la elaboración de las normas, por difícil que resulte el ejercicio, que permanecer como espectador mientras otros negocian normas. Si no son los miembros de la OMC los que desarrollan unos principios básicos, son los propios agentes privados quienes lo están haciendo sin gran dificultad aprovechando las fuertes economías de red existentes en la economía digital. Para el conjunto de miembros de la OMC sería preferible un conjunto de reglas, incluso muy senciIlas, pero que fueran de aplicación universal.

En definitiva, no es incompatible explotar en el corto plazo las flexibilidades previstas en los acuerdos de la OMC, con la fijación de un paquete de negociaciones futuras realista que, sin condicionantes previos, aborde no solo las cuestiones pendientes, como la protección industrial y agrícola, sino también los retos de la economía digital, más allá de la cuestión de la moratoria antes abordada. 